\title{
Finite Groups with Given Systems of $m$-S-Complemented Subgroups
}

\section{Khaled A. Al-Sharo ${ }^{1}$}

Received: 30 April 2019 / Revised: 13 July 2019 / Accepted: 18 July 2019 / Published online: 24 August 2019 (c) The Author(s) 2019

\begin{abstract}
Let $G$ be a finite group and $H$ a subgroup of $G$. We say that $H$ : is generalized $S$ quasinormal in $G$ if $H=\langle A, B\rangle$ for some modular subgroup $A$ and $S$-quasinormal subgroup $B$ of $G ; m$-S-complemented in $G$ if there are a generalized $S$-quasinormal subgroup $S$ and a subgroup $T$ of $G$ such that $G=H T$ and $H \cap T \leq S \leq H$. In this paper, we study finite groups with given systems of $m$-S-complemented subgroups. In particular, we prove that if $\mathfrak{F}$ is a saturated formation containing all supersoluble groups and $E$ is a normal subgroup of a finite group $G$ such that $G / E \in \mathfrak{F}$ and for every non-cyclic Sylow subgroup $P$ of $E$ every maximal subgroup of $P$ not having a nilpotent supplement in $G$ is $m$-S-complemented in $G$, then $G \in \mathfrak{F}$.
\end{abstract}

Keywords Finite group · Modular subgroup $\cdot S$-quasinormal subgroup · Generalized $S$-quasinormal subgroup $\cdot m$ - $S$-complemented subgroup

Mathematics Subject Classification 20D10 - 20D15 $\cdot 20$ D30

\section{Introduction}

Throughout this paper, all groups are finite and $G$ always denotes a finite group. Moreover, $\pi(G)$ is the set of all primes dividing the order $|G|$ of $G ; C_{n}$ denotes a cyclic group of order $n$.

Dedicated to the memory of Prof. L.A. Shemetkov.

Communicated by Mohammad Reza Darafsheh.

This paper is in final form and no version of it will be submitted for publication elsewhere.

Khaled A. Al-Sharo

sharo_kh@yahoo.com

1 Department of Mathematics, Al al-Bayt University, Mafraq 25113, Jordan 
A subgroup $M$ of $G$ is called modular in $G$ [1, p. 43] if (1) $\langle X, M \cap Z\rangle=\langle X, M\rangle \cap Z$ for all $X \leq G, Z \leq G$ such that $X \leq Z$, and (2) $\langle M, Y \cap Z\rangle=\langle M, Y\rangle \cap Z$ for all $Y \leq G, Z \leq G$ such that $M \leq Z$.

A subgroup $H$ of $G$ is said to be $S$-permutable [2,3] or $S$-quasinormal [4] in $G$ if $H$ permutes with every Sylow subgroup $P$ of $G$, that is, $H P=P H$. The subgroup $H$ of $G$ is said to be generalized $S$-quasinormal in $G$ [5] if there are a modular subgroup $A$ and an $S$-quasinormal subgroup $B$ of $G$ such that $H=\langle A, B\rangle$.

Interesting applications of generalized $S$-quasinormal subgroups were discussed in the paper [5]. In this paper, we consider the following generalization of such subgroups.

Definition 1.1 We say that a subgroup $H$ of $G$ is $m$-S-complemented in $G$ if there are a generalized $S$-quasinormal subgroup $S$ and a subgroup $T$ of $G$ such that $G=H T$ and $H \cap T \leq S \leq H$.

It is clear that every generalized $S$-quasinormal subgroup is $m-S$-complemented. Every modular subgroup and every $S$-quasinormal subgroup are generalized $S$ quasinormal. Now consider the following

Example 1.2 (1) Let $C_{3} 2 A_{4}=P \rtimes A_{4}$, where $A_{4}$ is the alternating group of degree 4 and $P$ is the base group of the regular wreath product $C_{3}$ ? $A_{4}$. Let $G=(P \rtimes$ $\left.A_{4}\right) \times\left(C_{11} \rtimes C_{5}\right)$, where $C_{11} \rtimes C_{5}$ is a non-abelian group of order 55. Let $Q$ be the Sylow 2-subgroup of $A_{4}$ and $R$ a Sylow 3-subgroup of $A_{4}$. Then, $P Q$ is supersoluble, so some subgroup $B$ of $P$ with $|B|=3$ is normal in $P Q$. Then, for every Sylow 3-subgroup $G_{3}$ of $G$ we have $B \leq P \leq G_{3}$, so $B G_{3}=G_{3}=G_{3} B$. On the other hand, for every Sylow 2-subgroup $Q^{x}$ of $G$ we have $Q^{x} \leq P Q$, so $B Q^{x}=Q^{x} B$. Hence, $B$ is $S$-quasinormal in $G$. In view of [1, Theorem 5.1.9], $A=C_{5}$ is modular in $G$. Then, $S=\langle A, B\rangle=A B$ is generalized $S$-quasinormal in $G$.

Now let $H=(A B) Q=A \times B Q$ and $T=P R C_{11}$. Then, $G=H T$ and $H \cap T=(A B) Q \cap P R C_{11}=B\left(A Q \cap P R C_{11}\right)=B \leq H$. Hence, $H$ is $m-S$ complemented in $G$.

Next, we show that $H$ is not generalized $S$-quasinormal in $G$. First note that $H_{G}=1$, so for every modular subgroup $V$ of $H$ we have $V^{G} \leq C_{11} \rtimes C_{5}$ by Lemma 2.4 below. Therefore, $A$ is the largest modular subgroup of $H$. Assume that $H$ is generalized $S$ quasinormal in $G$ and let $W$ be an $S$-quasinormal subgroup of $G$ such that $H=$ $\langle A, W\rangle=A W$. Then, $W_{G}=1$, so $W$ is a nilpotent subnormal subgroup of $G$ by [2, Theorem 1.2.17]. Hence for a Sylow 2-subgroup $Q_{1}$ of $W$, we have $1<Q_{1} \leq$ $O_{2}(G) \leq P \rtimes\left(Q \rtimes C_{p}\right)$ and so $Q_{1} \leq C_{G}(P)$, a contradiction. Therefore, $H$ is not generalized $S$-quasinormal in $G$.

(2) A subgroup $H$ of $G$ is said to be complemented (respectively, $c$-supplemented [6]) in $G$, if there is a subgroup $T$ of $G$ such that $G=H T$ and $H \cap T=1$ (respectively, $G=H T$ and $H \cap T \leq H_{G}$ ). It is clear that every complemented subgroup and every $c$-supplemented subgroup are $m$ - $S$-complemented.

(3) A subgroup $H$ of $G$ is said to be $S$-supplemented [7] (respectively, $m$ supplemented [8]) in $G$, if there are an $S$-quasinormal subgroup (respectively, a modular subgroup) $S$ and a subgroup $T$ of $G$ such that $G=H T$ and $H \cap T \leq S \leq H$. Every $S$-supplemented subgroup and every $m$-supplemented subgroup are $m$-Scomplemented. 
Let $K \leq H$ be normal subgroups of $G$. Then we say, following [1] that $H / K$ is hypercyclically embedded in $G$ if every chief factor of $G$ between $H$ and $K$ is cyclic. We say also that $H$ is hypercyclically embedded in $G$ if $H / 1$ is hypercyclically embedded in $G$.

Hypercyclically embedded subgroups play an important role in the theory of soluble groups (see the books [1-3]) and the conditions under which a normal subgroup is hypercyclically embedded were found by many authors (see, for example, the recent papers [9-17]).

In this paper, we prove the following results in this line research.

Theorem 1.3 Let $E$ be a normal subgroup of $G$ and let $P$ be a Sylow p-subgroup of $E$, where $p$ is the smallest prime dividing $|E|$. If every maximal subgroup of $P$ not having a p-nilpotent supplement in $G$ is $m$-S-complemented in $G$, then $E / O_{p^{\prime}}(E)$ is hypercyclically embedded in $G$.

Theorem 1.4 Let E be a normal subgroup of $G$. Suppose that for any Sylow subgroup $P$ of $E$ every maximal subgroup of $P$ not having a nilpotent supplement in $G$ is $m$-S-complemented in $G$. Then, E is hypercyclically embedded in $G$.

Recall that the formation $\mathfrak{F}$ is a homomorph of groups such that each group $G$ has the smallest normal subgroup (denoted by $G^{\mathfrak{F}}$ ) whose quotient is still in $\mathfrak{F}$. A formation $\mathfrak{F}$ is said to be saturated if $G \in \mathfrak{F}$ for any group $G$ with $G / \Phi(G) \in \mathfrak{F}$.

As a first application of Theorem 1.4, we prove also the following theorem which covers many known results (see Sect. 4 below).

Theorem 1.5 Let $\mathfrak{F}$ be a saturated formation containing all supersoluble groups, and let $X \leq E$ be normal subgroups of $G$ with $G / E \in \mathfrak{F}$. Suppose that for any Sylow subgroup $P$ of $X$ every maximal subgroup of $P$ not having a nilpotent supplement in $G$ is $m$-S-complemented in $G$. If $X=E$ or $X=F^{*}(E)$, then $G \in \mathfrak{F}$.

In this theorem, $X=F^{*}(E)$ denotes the generalized Fitting subgroup of $E$ [18, Ch. X], that is, the product of all normal quasinilpotent subgroups of $E$.

\section{Preliminaries}

The first lemma collects the properties of $S$-quasinormal subgroups used in our proofs.

Lemma 2.1 (See Chapter 1 in [2]). Let $A, B$ and $N$ be subgroups of $G$, where $A$ is $S$-quasinormal in $G$ and $N$ is normal in $G$.

(1) $A N / N$ is $S$-quasinormal in $G / N$.

(2) If $A \leq B$, then $A$ is $S$-quasinormal in $B$.

(3) If $N \leq B$ and $B / N$ is $S$-quasinormal in $G / N$, then $B$ is $S$-quasinormal in $G$.

(4) $A$ is subnormal in $G$ and $A^{G} / A_{G}$ is nilpotent.

(5) If $B$ is $S$-quasinormal in $G$, then $A \cap B$ and $\langle A, B\rangle$ are $S$-quasinormal in $G$.

Lemma 2.2 Let $A, B$ and $N$ be subgroups of $G$, where $A$ is generalized $S$-quasinormal in $G$ and $N$ is normal in $G$. Then 
(1) $A N / N$ is generalized $S$-quasinormal in $G / N$.

(2) If $A \leq B$, then $A$ is generalized $S$-quasinormal in $B$.

(3) If $N \leq B$ and $B / N$ is generalized $S$-quasinormal in $G / N$, then $B$ is generalized $S$-quasinormal in $G$.

(4) If $B$ is generalized $S$-quasinormal in $G$, then $\langle A, B\rangle$ is generalized $S$-quasinormal in $G$.

Proof Let $A=\langle L, T\rangle$, where $L$ is modular and $T$ is $S$-quasinormal subgroups of $G$.

(1) $A N / N=\langle L N / N, T N / N\rangle$, where $L N / N$ is modular in $G / N$ by Property (3) in [1, p. 201] and $T N / N$ is $S$-quasinormal in $G / N$ by Lemma 2.1(1). Hence, $A N / N$ is generalized $S$-quasinormal in $G / N$.

(2) This follows from Property (2) in [1, p. 201] and Lemma 2.1(2).

(3) Let $B / N=\langle V / N, W / N\rangle$, where $V / N$ is modular in $G / N$ and $W / N$ is $S$ quasinormal in $G / N$. Then, $B=\langle V, W\rangle$, where $V$ is modular in $G$ by Property (4) in [1, p. 201] and $W$ is $S$-quasinormal in $G$ by Lemma 2.1(3). Hence, $B$ is generalized $S$-quasinormal in $G$.

(4) This follows from Property (5) in [1, p. 201] and Lemma 2.1(5).

The lemma is proved.

Lemma 2.3 Let $A, B$ and $N$ be subgroups of $G$, where $A$ is $m$-S-complemented in $G$ and $N$ is normal in $G$.

(1) If either $N \leq A$ or $(|A|,|N|)=1$, then $A N / N$ is $m$-S -complemented in $G / N$.

(2) If $A \leq B$, then $A$ is $m$-S-complemented in $B$.

(3) If $N \leq B$ and $B / N$ is $m$-S-complemented in $G / N$, then $B$ is $m$-S-complemented in $G$.

Proof Let $T$ be a subgroup of $G$ such that $A T=G$ and $A \cap T \leq S \leq A$ for some generalized $S$-quasinormal subgroup $S$ of $G$. Then, $S=\langle L, M\rangle$, where $L$ is a modular and $M$ is an $S$-quasinormal subgroups of $G$.

(1) Note that $N T \cap N A=(T \cap A) N$. Indeed, if $N \leq A$, then $N T \cap N A=$ $N T \cap A=N(T \cap A)$. On the other hand, if $(|A|,|N|)=1$, then from $A T=G$ we get that $N \leq T$ and so $N T \cap N A=T \cap A N=N(T \cap A)$. Therefore, $G / N=(A N / N)(T N / N)$ and

$$
(A N / N) \cap(T N / N)=(A N \cap T N / N)=(A \cap T) N / N \leq S N / N,
$$

where $\mathrm{SN} / \mathrm{N}$ is a generalized $S$-quasinormal subgroup of $G / N$ by Lemma 2.2(1). Hence, $A N / N$ is $m$-S-supplemented in $G / N$.

(2) $B=A(B \cap T)$ and $(B \cap T) \cap A=T \cap A \leq S \leq A$, where $S$ is $m$ - $S$-permutable in $B$ by Lemma 2.2(2). Hence, $A$ is $m$-S-complemented in $B$.

(3) See the proof of (1) and use Lemma 2.2(3).

The lemma is proved.

Lemma 2.4 (See Theorem 5.2.5 in [1]). If $H$ is a modular subgroup of $G$, then $H^{G} / H_{G}$ is hypercyclically embedded in $G$. 
Lemma 2.5 (See Theorem 1.2 in [12]). If $E$ is a normal subgroup of $G$ and $F^{*}(E)$ is hypercyclically embedded in $G$, then $E$ is hypercyclically embedded in $G$.

Lemma 2.6 (See Lemma 2.16 in [7]). Suppose that $G / N \in \mathfrak{F}$, where $\mathfrak{F}$ is a saturated formation containing all supersoluble groups. If $N$ is hypercyclically embedded in $G$, then $G \in \mathfrak{F}$.

Lemma 2.7 (See Lemma 2.10 in [9]). Let $P$ be a Sylow p-subgroup of $G$, where $p$ is the smallest prime dividing $|G|$. If every maximal subgroup of $P$ has a p-nilpotent supplement in $G$, then $G$ is p-nilpotent.

Lemma 2.8 (See Lemma 2.12 in [19]). Let $P$ be a normal p-subgroup of $G$. If $P / \Phi(P)$ is hypercyclically embedded in $G$, then $P$ is hypercyclically embedded in $G$.

\section{Proofs of Theorems 1.3, 1.4 and 1.5}

The product of all hypercyclically embedded subgroups of $G$ is denoted by $Z_{\mathfrak{U}}(G)$ and it is called the supersoluble hypercentre of $G$. Note that if $A$ and $B$ are normal hypercyclically embedded subgroups of $G$, then (in view of the $G$-isomorphism $A B / A \simeq B /(B \cap A))$ the product $A B$ is also hypercyclically embedded in $G$.

Proof of Theorem 1.3. Suppose that this theorem is false and consider a counterexample $(G, E)$ for which $|G|+|E|$ is minimal. Then, $G$ is not supersoluble. Let $Z=Z_{\mathfrak{U}}(G)$.

(1) If $R$ is a minimal normal subgroup of $G$ and $R$ is either a $p^{\prime}$-group or a $p$-subgroup contained in $E$ such that $R \neq P$, then the hypothesis holds for $(G / R, E R / R)$.

First, we show that $P R / R$ is a Sylow $p$-subgroup of $E R / R$. Indeed, $P \cap R$ is a Sylow $p$-subgroup of $R$ and $P R / R \simeq P /(P \cap R)$ is a $p$-subgroup of $E R / R$. On the other hand, from

$$
\begin{aligned}
|(E R / R):(P R / R)| & =|E R: P R|=|E||R||P \cap R|:|E \cap R||P||R| \\
& =|E||P \cap R|:|E \cap R||P|
\end{aligned}
$$

we get that $|E R / R: P R / R|$ is a $p^{\prime}$ - number since the minimality of $R$ implies that we have either $R \cap E=1$ or $E \cap R=R$. Therefore $P R / R$ is a Sylow $p$-subgroup of $E R / R$.

Now let $V / R$ be a maximal subgroup of $P R / R$. Then, $V=(V \cap P) R$ and

$$
\begin{aligned}
p & =|(P R / R):(V / R)|=|P R:(V \cap P) R| \\
& =|P||R||(V \cap P) \cap R|:|P \cap R||V \cap P||R| \\
& =|P||V \cap R|:|P \cap R||V \cap P| .
\end{aligned}
$$

First, suppose that $R$ is a $p^{\prime}$-group. Then, $p=|P||V \cap R|:|P \cap R||V \cap P|=\mid P$ : $V \cap P \mid$, so $V \cap P$ is a maximal subgroup of $P$. Then, by hypothesis, either $V \cap P$ has a $p$-nilpotent supplement $S$ in $G$ or $V \cap P$ is $m$-S-complemented in $G$. In the first 
case, $S R / R \simeq S /(S \cap R)$ is a $p$-nilpotent supplement of $V / R=(V \cap P) R / R$ in $G / R$. In the second case, $V / R$ is $m$-S-complemented in $G / R$ by Lemma 2.3(1). Now suppose that $R$ is a $p$-subgroup contained in $E$. Then, $R \leq P$ and so $p=\mid(P R / R)$ : $(V / R)|=| P: V \mid$. Then, by hypothesis, either $V$ has a $p$-nilpotent supplement $S$ in $G$ or $V$ is $m$-S-complemented in $G$. Therefore, $V / R$ has a $p$-nilpotent supplement $S R / R$ in $G / R$ or $V / R$ is $m$-S-complemented in $G / R$ by Lemma 2.3(1). Hence, the hypothesis folds for $(G / R, E R / R)$.

(2) If $H / K$ is a chief factor of $E$ below $E$ and $|H / K|=p$, then $C_{E}(H / K)=E$.

Since $p$ is the smallest prime dividing $|E|$ by hypothesis, this follows from the fact that $E / C_{E}(H / K) \simeq V \leq \operatorname{Aut}(H / K)$ and from the fact that $\operatorname{Aut}(A)$ is a cyclic group of order $p-1$ for any group $A$ of order $p$.

(3) If $R$ is a minimal normal subgroup of $G$ and $R$ is either a $p^{\prime}$-group or a $p$ subgroup contained in $E$ such that $R \neq P$, then $E R / R$ is $p$-nilpotent and $(E R / R) / O_{p^{\prime}}(E R / R)$ is hypercyclically embedded in $G / R$.

The hypothesis holds for $(G / R, E R / R)$ by Claim $(1)$, so $(E R / R) / O_{p^{\prime}}(E R / R)$ is hypercyclically embedded in $G / R$ by the choice of $G$. Therefore, $E R / R$ is $p$-nilpotent by Claim (2).

(4) $O_{p^{\prime}}(G)=1$.

Assume that $O_{p^{\prime}}(G) \neq 1$ and let $R$ be a minimal normal subgroup of $G$ contained in $O_{p^{\prime}}(G)$. Then, $(E R / R) / O_{p^{\prime}}(E R / R)$ is hypercyclically embedded in $G / R$ and $E R / R \simeq E / E \cap R$ is $p$-nilpotent by Claim (3). Hence, $E$ is $p$-nilpotent and from

$$
(E R / R) / O_{p^{\prime}}(E R / R)=(E R / R) /\left(O_{p^{\prime}}(E R) / R\right)=(E R / R) /\left(O_{p^{\prime}}(E) R / R\right)
$$

and from the $G$-isomorphisms

$$
\begin{aligned}
& (E R / R) /\left(O_{p^{\prime}}(E) R / R\right) \simeq E R / O_{p^{\prime}}(E) R \simeq E / E \cap O_{p^{\prime}}(E) R \\
& \quad=E / O_{p^{\prime}}(E)(E \cap R)=E / O_{p^{\prime}}(E)
\end{aligned}
$$

we get that $E / O_{p^{\prime}}(E)$ is hypercyclically embedded in $G$, contrary to the choice of $(G, E)$. Hence, we have (4).

(5) $Z \cap E \leq Z_{\infty}(E)$.

Since $Z$ is clearly supersoluble, a Sylow $q$-subgroup $Q$ of $Z$, where $q$ is the largest prime dividing $|Z|$, is normal and so characteristic in $Z$. Then, $Q$ is normal in $G$, which implies that $Z=Q$ and $q=p$ by Claim (4), so $Z \cap E \leq Z_{\infty}(E) \leq P$ since $p$ is the smallest prime dividing $E$ by hypothesis.

(6) $P \neq R$ for each minimal normal subgroup $R$ of $G$.

Assume that $P=R$ and let $V$ be any maximal subgroup of $P$. Then, by hypothesis, either $V$ has a $p$-nilpotent supplement $S$ in $G$ or $V$ is $m$-S-complemented in $G$. In the former case, we have $S \neq G$ since $G$ is not $p$-nilpotent. On the other hand, in this case, we have $P=V(P \cap T)$, where $P \cap T$ is clearly normal in $G$ and so the minimality of $R=P$ implies that $P \cap T=1$. But then $V=P$. This contradiction shows that $V$ is $m$-S-complemented in $G$, so there are an $m$-S-permutable subgroup $S$ and a subgroup $T$ of $G$ such that $G=V T$ and $V \cap T \leq S \leq V$. Let $A$ be a modular subgroup and $B$ an $S$-quasinormal subgroup 
of $G$ such that $S=\langle A, B\rangle$. Then, $A_{G}=1$, so $A^{G} \leq Z$ by Lemma 2.4. Therefore, $A=1$ and so $S=B$ is $S$-quasinormal in $G$. But then $S$ is normal in $G$ by Lemma 1.2.16 in [2]. Hence, $S=1$ and so $T \cap V=1$. But then $1<T \cap R<R$, where $T \cap R$ is normal in $G$. This contradiction shows that we have (6).

(7) If $M$ is a proper subgroup of $G$ containing $E$, then $E / O_{p^{\prime}}(E)$ is hypercyclically embedded in $M$. Hence $E=P$.

Let $V$ be a maximal subgroup of $P$. Then, either $V$ has a $p$-nilpotent supplement $S$ in $G$ or $V$ is $m$-S-complemented in $G$. In the former case, we have $M=V(M \cap S)$, so $M \cap S$ is a $p$-nilpotent supplement to $V$ in $G$. In the second case, the subgroup $V$ is $m$-S-complemented in $M$ by Lemma 2.3(2). Hence, the hypothesis holds for $(M, E)$, so $E / O_{p^{\prime}}(E)$ is hypercyclically embedded in $M$ by the choice of $G$. Claim (2) implies that $E$ is $p$-nilpotent. On the other hand, $O_{p^{\prime}}(E)$ is characteristic in $E$ and so it is normal in $G$. Then, $O_{p^{\prime}}(E) \leq O_{p^{\prime}}(G)=1$ by Claim (4). Therefore, $E$ is supersoluble, which implies that a Sylow $q$-subgroup $Q$ of $E$, where $q$ is the largest prime dividing $|E|$, is normal and hence characteristic in $E$. Hence, $q=p$ and $E=P=Q$ by Claim (4).

(8) $E$ is $p$-nilpotent.

Assume that this is false. Then, $E \neq P$, so $E=G$ by Claim (7).

(a) $O_{p}(G) \neq 1$.

Assume that $O_{p}(G)=1$. Lemma 2.7 implies that some maximal subgroup $V$ of $P$ has no $p$-nilpotent supplement in $G$, so $V$ is $m$-S-complemented in $G$. Then, there are a generalized $S$-quasinormal subgroup $S$ and a subgroup $T$ of $G$ such that $G=V T$ and $V \cap T \leq S \leq V$. Let $A$ be a modular subgroup and $B$ an $S$-quasinormal subgroup of $G$ such that $S=\langle A, B\rangle$. Then, $B P^{x}=P^{x} B=P^{x}$ for all $x \in G$, so $B \leq P_{G}=O_{p}(G)=1$. Hence, $S=A$ and $A_{G}=1$; therefore, $S \leq Z \leq Z_{\infty}(G)$ by Lemma 2.4 and Claim (5) since $E=G$.

Since $Z_{\infty}(G)$ is nilpotent, a Sylow $p$-subgroup of $Z_{\infty}(G)$ is normal in $G$, so $A=S=1$ since $V_{G}=1$. Therefore, $T$ is a complement to $V$ in $G$, so for a Sylow $p$-subgroup $T_{p}$ of $T$ we have $\left|T_{p}\right|=p$. Therefore, $T$ is $p$-nilpotent by [20, IV, 2.8]. Hence, every maximal subgroup $V$ of $P$ has a $p$-nilpotent complement in $G$, so $G$ is $p$-nilpotent by Lemma 2.7. This contradiction shows that we have (a).

(b) $O_{p}(G)=C_{G}\left(O_{p}(G)\right)$ is a minimal normal subgroup of $G$ and $O_{p}(G) \not \leq$ $\Phi(G)$.

By Claim (a), $O_{p}(G) \neq 1$. Let $R$ be a minimal normal subgroup of $G$ contained in $O_{p}(G)$. Then, $G / R$ is $p$-nilpotent by Claims (3) and (6). Hence, $G$ is $p$-soluble. Therefore, every minimal normal subgroup $R$ of $G$ is a $p$-group by Claim (2). Hence, $R$ is the unique minimal normal subgroup of $G$ and $R \not \leq \Phi(G)$, so $R=C_{G}(R)=O_{p}(G)$ by [33, Ch. A, 15.6]. It is clear also that $|R|>p$, so $Z=1$.

Final contradiction for (8).

Let $V$ be any maximal subgroup of $P$. We show that $V$ has a $p$-nilpotent supplement in $G$. Assume that this is false. Then, the subgroup $V$ is $m-S$-complemented in $G$ by hypothesis.

First suppose that $R \not \leq V$. Then, $W=V \cap R$ is normal in $P,|R: W|=p$ and, by Claim (b), $V_{G}=1$. There are an generalized $S$-quasinormal subgroup $S$ and a subgroup $T$ of $G$ such that $G=V T$ and $V \cap T \leq S \leq V$. Then, $V \cap T=S \cap T$. Arguing 
as above, we can show that $S$ is $S$-quasinormal in $G$. Hence, $S$ is subnormal in $G$ by Lemma 2.1(4). It follows that $S \leq O_{p}(G)=R$ by Claim (b). Hence, $S \leq R \cap V=W$ and so $S^{G}=S^{P O^{p}(G)}=S^{W} \leq W$ by [2, Lemma 1.2.16], which implies that $S=1$. Then, $T$ is a complement to $V$ in $G$, so $T$ is $p$-nilpotent.

Now let $V$ be any maximal subgroup of $P$ containing $R$, and let $M$ be a maximal subgroup of $G$ such that $G=R \rtimes M$. Then, $M \simeq G / R$ is $p$-nilpotent, so $M$ is a $p$ nilpotent supplement to $V$ in $G$. Thus, every maximal subgroup of $P$ has a $p$-nilpotent supplement in $G$. Therefore, $G$ is $p$-nilpotent by Lemma 2.7. This contradiction shows that we have (8).

The final contradiction. Claims (2) and (8) imply that $E=P$ is a normal $p$ subgroup of $G$. Let $R$ be a minimal normal subgroup of $G$ contained in $P$. Then, $P / R$ is hypercyclically embedded in $G$ by Claims (3) and (6). Therefore, $R \not \leq \Phi(P)$ by Lemma 2.8 and [20, III, Hilfsatz 3.3(a)]. Hence, $\Phi(P)=1$, so $P$ is an elementary abelian $p$-group. If $|R|=p$, then $P$ is hypercyclically embedded in $G$ by the JordanHölder theorem for the chief series. Hence, $R$ is not cyclic. Moreover, $R$ is the unique minimal normal subgroup of $G$ contained in $P$. Indeed, suppose that for some minimal normal subgroup $N \neq R$ of $G$ we also have $N \leq P$. Then, $P / N$ is hypercyclically embedded in $G$ and so from the $G$-isomorphism $R N / N \simeq R$ we get that $|R|=p$, a contradiction.

Let $W$ be a maximal subgroup of $N$ such that $W$ is normal in a Sylow $p$-subgroup $G_{p}$ of $G$. Then, $W \neq 1$. We show that $W$ is $S$-quasinormal in $G$. Let $B$ be a complement to $N$ in $P$ and $H=W B$. Then, $H$ is a maximal subgroup of $P$ and $W=H \cap R$. Therefore, $W$ is $S$-quasinormal in $G$ in the case when $H$ is $S$-quasinormal in $G$ by Lemma 2.1(5). From now on, we suppose that $H$ is not $S$-quasinormal in $G$.

Assume that $H$ has a $p$-nilpotent supplement $U$ in $G$ and let $S$ be the normal $p$ complement in $U$. Then, $P=P \cap H U=H(P \cap U)$, where $P \cap U$ is normal in $G$ since $P$ is abelian. Moreover, $1<P \cap U<P$ since $G$ is not $p$-nilpotent. Therefore, $R \leq P \cap U$. Then, $[R, S]=1$, so $G / C_{G}(R)$ is a $p$-group and so $C_{G}(R)=G$ since $R$ is a $p$-group. But then $|R|=p$. This contradiction shows that $H$ has no $p$-nilpotent supplements in $G$ and hence $H$ is $m$-S-complemented in $G$ by hypothesis.

Let $S$ and $T$ be subgroups of $G$ such that $S$ is generalized $S$-quasinormal in $G$ and we have $G=H T$ and $H \cap T \leq S \leq H$. And let $S=A B$, where $A$ is modular and $B$ is $S$-quasinormal in $G$. Then, $N \not \leq H$ and so $A_{G}=1$, which implies that $A^{G}$ is hypercyclically embedded in $G$ by Lemma 2.4 . But then $A=1$ since otherwise $N \leq A^{G} \cap P$ and so $|N|=p$. Therefore, $S=B$ is $S$-quasinormal in $G$. Since $T \cap H \leq S \leq H$ and $H$ is not $S$-quasinormal in $G$, it follows that $T<G$ and for the normal subgroup $T \cap P$ of $G$ we have $1<T \cap P$. Then, $N \leq T$ and so $N \cap H=N \cap S=W$, which implies that $W$ is $S$-quasinormal in $G$ by Lemma 2.1(5). But then $W$ is normal in $G$ since $G=G_{p} O^{p}(G) \leq N_{G}(W)$ by [2, Lemma 1.2.16] and so $W=1$. Therefore, $N$ is cyclic. This contradiction completes the proof of the result.

Proof of Theorem 1.4. Suppose that this theorem is false and consider a counterexample $(G, E)$ for which $|G|+|E|$ is minimal. Let $p$ be the smallest prime dividing $|E|$ and let $P$ be a Sylow $p$-subgroup of $E$. 
Then, $E$ is $p$-supersoluble by Theorem 1.3 and so $E$ is $p$-nilpotent since $p$ is the smallest prime dividing $|E|$ (see Claim (2) in the proof of Theorem 1.3). Note also that if $X$ is a non-identity Hall subgroup of $E$, then $X=E$. Indeed, the hypothesis holds for $(G / X, E / X)$ and for $(G, X)$ by Lemma 2.3(1). Hence in the case $X \neq E$, the choice of $G$ implies that $E / X$ and $X$ are hypercyclically embedded in $G$. Hence, $E$ is hypercyclically embedded in $G$ by the Jordan-Hölder theorem for the chief series. This contradiction shows that $E=P$, so $E$ is hypercyclically embedded in $G$ by Theorem 1.3. The theorem is proved.

Proof of Theorem 1.5. This theorem is a corollary of Theorem 1.4 and Lemmas 2.5 and 2.6.

\section{Some Applications of the Results}

Theorems 1.3, 1.4 and 1.5 cover many known results. In particular, from Theorem 1.5, we get the following known results.

Corollary 4.1 (Srinivasan [21]). If the maximal subgroups of the Sylow subgroups of $G$ are $S$-quasinormal in $G$, then $G$ is supersoluble.

Corollary 4.2 (Asaad [22]). Let $\mathfrak{F}$ be a saturated formation containing all supersoluble groups and $G$ a group with a normal subgroup $E$ such that $G / E \in \mathfrak{F}$. If $G / E \in \mathfrak{F}$ and every maximal subgroup of every Sylow subgroup of $E$ is $S$-quasinormal in $G$, then $G \in \mathfrak{F}$.

A subgroup $H$ of $G$ is said to be $c$-normal in $G$ [23], if there is a normal subgroup $T$ of $G$ such that $G=H T$ and $H \cap T \leq H_{G}$. It is clear that every $c$-normal subgroup of $G$ is also $m$-S-complemented in $G$. Hence, we get from Theorem 1.5 the following known results.

Corollary 4.3 (Wang [23]). If the maximal subgroups of the Sylow subgroups of G are c-normal in $G$, then $G$ is supersoluble.

Corollary 4.4 (Alsheik Ahmad [24]). If the maximal subgroups of the Sylow subgroups of $G$ not having supersoluble supplement in $G$ are c-normal in $G$, then $G$ is supersoluble.

Corollary 4.5 (Ramadan [25]). Let $E$ be a normal subgroup of $G$ with supersoluble quotient $G / E$. If all maximal subgroups of the Sylow subgroups of E are normal in $G$, then $G$ is supersoluble.

Corollary 4.6 (Li, Guo [26]). Let $E$ be a soluble normal subgroup of $G$ with supersoluble quotient $G / E$. If all maximal subgroups of the Sylow subgroups of $F(E)$ are c-normal in $G$, then $G$ is supersoluble.

Corollary 4.7 (Wey [27]). Let $\mathfrak{F}$ be a saturated formation containing all supersoluble groups and $G$ a group with a soluble normal subgroup $E$ such that $G / E \in \mathfrak{F}$. If all maximal subgroups of the Sylow subgroups of $F(E)$ are c-normal in $G$, then $G \in \mathfrak{F}$. 
Corollary 4.8 (Wei, Wang, Li [28]). Let $\mathfrak{F}$ be a saturated formation containing all supersoluble groups and $G$ a group with a normal subgroup $E$ such that $G / E \in \mathfrak{F}$. If all maximal subgroups of the Sylow subgroups of $F^{*}(E)$ are c-normal in $G$, then $G \in \mathfrak{F}$.

Corollary 4.9 (Asaad, Ramadan, Shaalan [29]). Let E be a soluble normal subgroup of $G$ with supersoluble quotient $G / E$. Suppose that all maximal subgroups of any Sylow subgroup of $F(E)$ are $S$-quasinormal in $G$. Then, $G$ is supersoluble.

Corollary 4.10 (Li, Wang [30]). Let $\mathfrak{F}$ be a saturated formation containing all supersoluble groups and $G$ a group with a normal subgroup $E$ such that $G / E \in \mathfrak{F}$. If all maximal subgroups of any Sylow subgroup of $F^{*}(E)$ are $S$-quasinormal in $G$, then $G \in \mathfrak{F}$.

Corollary 4.11 (Li, Wang [30]). Let $\mathfrak{F}$ be a saturated formation containing all supersoluble groups and $G$ a group with a normal subgroup $E$ such that $G / E \in \mathfrak{F}$. If every maximal subgroup of every Sylow subgroup of $F^{*}(E)$ is $S$-quasinormal in $G$, then $G \in \mathfrak{F}$.

Corollary 4.12 (Wei [28]). Let $\mathfrak{F}$ be a saturated formation containing all supersoluble groups and $G$ a group with a normal subgroup $E$ such that $G / E \in \mathfrak{F}$. If every maximal subgroup of every Sylow subgroup of $E$ is c-normal in $G$, then $G \in \mathfrak{F}$.

In view of Example 1.2(ii), we get also from Theorem 1.5 the following known results.

Corollary 4.13 (Wei, Wang and $\mathrm{Li}[31]$ ). Let $\mathfrak{F}$ be a saturated formation containing all supersoluble groups and $G$ a group with a normal subgroup $E$ such that $G / E \in \mathfrak{F}$. If every maximal subgroup of every Sylow subgroup of $F^{*}(E)$ is c-supplemented in $G$, then $G \in \mathfrak{F}$.

Corollary 4.14 (Ballester-Bolinches and Guo [32]). Let $\mathfrak{F}$ be a saturated formation containing all supersoluble groups and $G$ a group with a normal subgroup $E$ such that $G / E \in \mathfrak{F}$. If every maximal subgroup of every Sylow subgroup of $E$ is c-supplemented in $G$, then $G \in \mathfrak{F}$.

Acknowledgements The author would like to thank the referee for his/her comments and suggestions to improve the manuscript.

Open Access This article is distributed under the terms of the Creative Commons Attribution 4.0 International License (http://creativecommons.org/licenses/by/4.0/), which permits unrestricted use, distribution, and reproduction in any medium, provided you give appropriate credit to the original author(s) and the source, provide a link to the Creative Commons license, and indicate if changes were made.

\section{References}

1. Schmidt, R.: Subgroup Lattices of Groups. Walter de Gruyter, Berlin (1994)

2. Ballester-Bolinches, A., Esteban-Romero, R., Asaad, M.: Products of Finite Groups. Walter de Gruyter, Berlin (2010) 
3. Guo, W.: Structure Theory for Canonical Classes of Finite Groups. Springer, Berlin (2015)

4. Kegel, O.H.: Sylow-Gruppen und subnormalteiler endlicher Gruppen. Math. Z. 78, 205-221 (1962)

5. Hu, B., Huang, J., Skiba, A.N.: On generalized $S$-quasinormal and generalized subnormal subgroups of finite groups. Commun. Algebra 46(4), 1758-1769 (2018)

6. Ballester-Bolinches, A., Wang, Y., Guo, X.Y.: $c$-supplemented subgroups of finite groups. Glasgow Math. J. 42, 383-389 (2000)

7. Skiba, A.N.: On weakly $s$-permutable subgroups of finite groups. J. Alegebra 315, 192-209 (2007)

8. Vasil'ev, V.A., Skiba, A.N.: On one generalization of modular subgroups. Ukrain. Math. J. 63(10), 1494-1505 (2012)

9. Shemetkov, L.A., Skiba, A.N.: On the $\mathfrak{X} \Phi$-hypercentre of finite groups. J. Algebra 322, 2106-2117 (2009)

10. Asaad, M.: Finite groups with certain subgroups of Sylow subgroups complemented. J. Algebra 323, 1958-1965 (2010)

11. Li, B.: On П-property and П-normality of subgroups of finite groups. J. Algebra 334, 321-337 (2011)

12. Skiba, A.N.: On two questions of L.A. Shemetkov concerning hypercyclically embedded subgroups of finite groups. J. Group Theory 13, 841-850 (2010)

13. Skiba, A.N.: A characterization of the hypercyclically embedded subgroups of finite groups. J. Pure Appl. Algebra 215, 257-261 (2011)

14. Skiba, A.N.: Cyclicity conditions for $G$-chief factors of normal subgroups of a group $G$. Sib. Math. J. 52(3), 127-130 (2011)

15. Guo, W., Skiba, A.N.: Finite groups with permutable complete Wielandt sets of subgroups. J. Group Theory 18, 191-200 (2015)

16. Yi, X., Skiba, A.N.: Some new characterizations of PST-groups. J. Algebra 399, 39-54 (2014)

17. Hu, B., Huang, J., Skiba, A.N.: On weakly $\sigma$-quasinormal subgroups of finite groups. Publ. Math. Debrecen 92(1-2), 201-216 (2018)

18. Huppert, B., Blackburn, N.: Finite Groups III. Springer, Berlin (1982)

19. Chen, X., Guo, W., Skiba, A.N.: Some conditions under which a finite group belongs a Baer local formation. Commun. Algebra 42, 4188-4205 (2014)

20. Huppert, B.: Endliche Gruppen I. Springer, Berlin (1967)

21. Srinivasan, S.: Two sufficient conditions for supersolvability of finite groups. Israel J. Math. 35, 210214 (1980)

22. Asaad, M.: On maximal subgroups of finite group. Commun. Algebra 26, 3647-3652 (1998)

23. Wang, Y.: c-normality of groups and its properties. J. Algebra 180, 954-965 (1996)

24. Ahmad, A.Alsheik: Finite groups with given $c$-permutable subgroups. Algebra Discrete Math. 2, 9-16 (2004)

25. Ramadan, M.: Influence of normality on maximal subgroups of Sylow subgroups of a finite group. Acta Math. Hung. 59, 107-110 (1992)

26. Li, D., Guo, X.: The influence of $c$-normality of subgroups on the structure of finite groups, II. Commun. Algebra 26, 1913-1922 (1998)

27. Wei, H.: On $c$-normal maximal and minimal subgroups of Sylow subgroups of finite groups. Commun. Algebra 29, 2193-2200 (2001)

28. Wei, H., Wang, Y., Li, Y.: On $c$-normal maximal and minimal subgroups of Sylow subgroups of finite groups. II. Commun. Algebra 31, 4807-4816 (2003)

29. Asaad, M., Ramadan, M., Shaalan, A.: Influence of $\pi$-quasinormality on maximal subgroups of Sylow subgroups of fitting subgroup of a finite group. Arch. Math. 56, 521-527 (1991)

30. Li, Y., Wang, Y.: The influence of $\pi$-quasinormality of some subgroups of a finite group. Arch. Math. (Basel) 81, 245-252 (2003)

31. Wang, Y., Wei, H., Li, Y.: A generalization of Kramer's theorem and its applications. Bull. Aust. Math. Soc. 65, 467-475 (2002)

32. Ballester-Bolinches, A., Guo, X.Y.: On complemented subgroups of finite groups. Arch. Math. 72, 161-166 (1999)

33. Doerk, K., Hawkes, T.: Finite Soluble Groups. Walter de Gruyter, Berlin (1992)

Publisher's Note Springer Nature remains neutral with regard to jurisdictional claims in published maps and institutional affiliations. 